\title{
CHAPITRE 15 : OBSESSION DE RÉUSSITE ET AGRESSIONS À L'ÉCOLE
}

Alain Clémence

in Fabrizio Butera et al., L'évaluation, une menace?

Presses Universitaires de France | « Apprendre »

2011 | pages 157 à 165

ISBN 9782130589600

Article disponible en ligne à l'adresse :

http://www.cairn.info/l-evaluation-une-menace--9782130589600-page-157.htm

\section{!Pour citer cet article :}

Alain Clémence, «Chapitre 15: Obsession de réussite et agressions à l'école », in Fabrizio Butera et al., L'évaluation, une menace?, Presses Universitaires de France « Apprendre », 2011 (), p. 157-165.

DOI 10.3917/puf.darno.2011.01.0157

Distribution électronique Cairn.info pour Presses Universitaires de France.

(C) Presses Universitaires de France. Tous droits réservés pour tous pays.

La reproduction ou représentation de cet article, notamment par photocopie, n'est autorisée que dans les limites des conditions générales d'utilisation du site ou, le cas échéant, des conditions générales de la licence souscrite par votre établissement. Toute autre reproduction ou représentation, en tout ou partie, sous quelque forme et de quelque manière que ce soit, est interdite sauf accord préalable et écrit de l'éditeur, en dehors des cas prévus par la législation en vigueur en France. Il est précisé que son stockage dans une base de données est également interdit. 


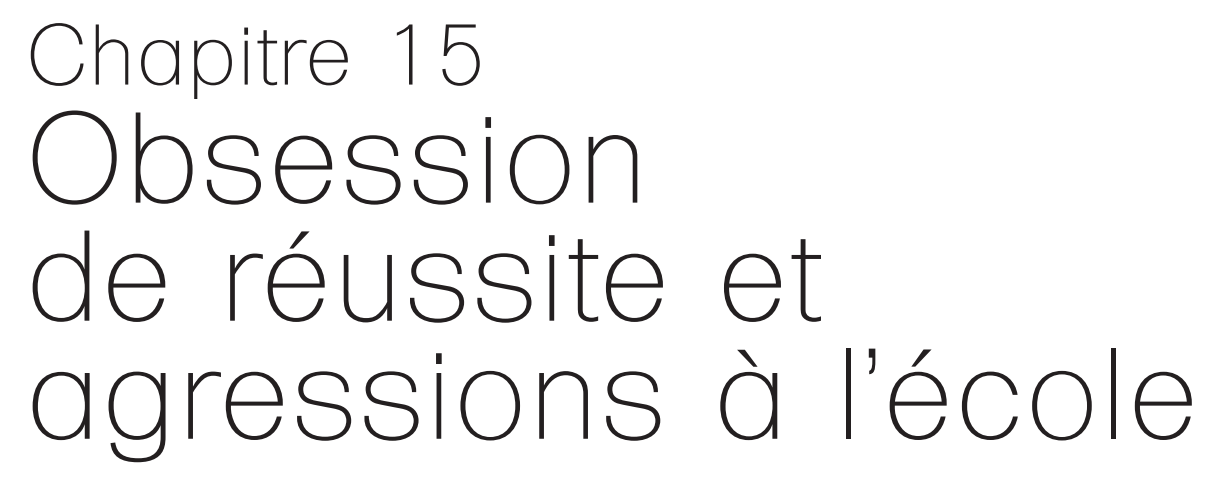

Alain Clémence

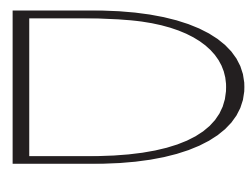

ébutons par une question que nous entendons souvent autour des écoles : les mauvais élèves commettent-ils davantage d'agressions que les bons élèves ?

La réponse intuitive est souvent positive. Dans ce chapitre, nous présentons une approche de la question en cheminant dans une littérature scientifique abondante et passionnante. Nous abordons d'abord le sens de la relation entre les performances scolaires et les manifestations agressives, une relation qui s'avère plus complexe que prévu. Ensuite, nous évoquons deux approches théoriques qui permettent finalement d'éclairer et de mieux comprendre cette relation complexe.

\section{L'agressivité favorise-t-elle la réussite scolaire?}

Cette question, presque incongrue aujourd'hui, est celle que posent Wyer, Weatherley et Terrel dans une recherche publiée en $1965^{1}$. Des étudiants universitaires remplissaient un questionnaire susceptible de définir leur « hostilité manifeste » composée principalement de la réalisation d'actes violents, de l'expression de pensées agressives et 
L'approche interactionniste de la déviance des années 1960 met l'accent sur les déterminants sociaux au détriment des composantes individuelles. de l'absence de culpabilité face à l'agression. Leur performance académique était mesurée par la moyenne de leurs résultats effectifs et un score global à un test d'aptitudes verbales et mathématiques. Les résultats montrent que la réussite académique est plus élevée chez les hommes qui manifestent le plus d'agressions et éprouvent le moins de culpabilité, alors que chez les femmes, la performance est meilleure lorsqu'elles expriment moins de pensées agressives et davantage de culpabilité. Les auteurs expliquent la différence entre les hommes et les femmes par leurs rôles sociaux. Les étudiants se préparent à occuper des positions dominantes dont l'accès requiert des dispositions agressives et compétitives. Les hommes les mieux préparés pour occuper ces rôles sociaux sont ceux qui parviennent à exprimer clairement leurs tendances agressives de manière directe, ou de manière indirecte dans le cadre de la compétition académique. Par conséquent, ces individus agressifs et désinhibés obtiennent de meilleurs résultats que leurs camarades aux prises avec des réserves émotionnelles qui limitent la manifestation d'actes agressifs. L'absence de culpabilité face à de tels comportements facilite leur manifestation effective et la reconnaissance des dispositions à exercer un rôle dominant. À l'inverse, les principaux rôles dévolus aux femmes dans la société, ceux de ménagère ou de mère, incitent à adopter une position soumise pour éviter les situations susceptibles de menacer la domination masculine ${ }^{2}$. Les femmes doivent de ce fait contrôler leurs « besoins agressifs » et les dériver vers des domaines propices à la réalisation d'autres buts, comme la réussite académique. Une culpabilité élevée associée à une faible expression d'hostilité conduit donc les étudiantes à obtenir une meilleure réussite académique.

Les résultats et le raisonnement exposés dans cette recherche offrent une excellente ouverture pour répondre à notre question, même si la définition des rôles sociaux apparaît un brin datée. Autant dire que les études conduites dans ce domaine sont aussi façonnées par le contexte social et politique qui les encadre. Ainsi, cette étude menée par des psychologues s'inscrit dans le développement d'une approche interactionniste de la déviance dans les années 1960, approche qui met l'accent sur les déterminants sociaux au détriment des composantes individuelles. Mais cette perspective va progressivement changer avec la conduite de grandes études, longitudinales dès les années 1970. En par- 
ticulier, le sens de la causalité entre performance scolaire et agression est rediscutée, avec l'idée que la déviance, sous la forme de comportements violents ou délinquants, serait produite par la faiblesse des capacités intellectuelles.

\section{La violence est-elle créée par un faible quotient intellectuel ?}

Étonnamment, c'est un texte publié en 1977 par deux sociologues, Hirschi et Hindelang, qui va mener une attaque en règle contre les travaux sociologiques sur la déviance ${ }^{3}$. Selon les auteurs, ces travaux auraient totalement occulté des résultats empiriques en cherchant à gommer l'impact de facteurs individuels, et en particulier celui du quotient intellectuel (QI) sur les comportements agressifs et plus généralement délinquants. Ils rapportent quelques études qui montrent une corrélation négative entre le QI et la réalisation d'actes délinquants et violents. Dans leur revue de la littérature, très polémique, ils discutent et éliminent des biais qui sont attribués à la mesure du QI et de la délinquance. Ainsi, ils relèvent que certes le QI est associé à la classe sociale, mais son impact reste déterminant même lorsque l'appartenance sociale ou ethnique est contrôlée. De même, ils réfutent l'argument selon lequel les personnes les plus intelligentes commettent autant d'actes répréhensibles que les autres, mais qu'elles évitent plus aisément les arrestations policières ou les condamnations pénales et déclarent moins d'actes répréhensibles dans les enquêtes. Bien entendu, la délinquance ne se réduit pas aux agressions, ni ne les couvre toutes. Néanmoins, ce texte est sinon le déclencheur, du moins un révélateur d'un courant de recherches qui va explorer plus intensivement la violence dans le champ scolaire. Les liens de causalité entre violence et performance scolaire vont apparaître progressivement plus complexes.

\section{L'hyperactivité est-elle la cause de la violence et de l'échec scolaire?}

Le nombre d'études sur l'explication des difficultés scolaires, autant comportementales que cognitives, explose littéralement à la fin des années 1970. En 1992, Hinschaw 
Les antécédents à la période scolaire peuvent expliquer à la fois le niveau des comportements répréhensibles et celui des résultats scolaires. publie une revue de 20 études longitudinales ${ }^{4}$, réalisés entre 1970 et 1985, pour tenter d'apporter une réponse aux liens de causalité entre la performance scolaire et des comportements problématiques manifestant de l'impulsivité, de l'agressivité, de la défiance ou de la suractivité. L'analyse des résultats distingue les niveaux d'études primaire et secondaire. Relevons qu'un tiers des études ne concernent que les garçons, ce taux dépassant la moitié pour les adolescents. Concernant ces derniers, les recherches mettent clairement en évidence un lien négatif entre les comportements problématiques, et notamment les agressions, et la réussite scolaire. Cependant, aucune conclusion claire ne peut être tirée sur la direction causale. Une illustration exemplaire est fournie par une grande recherche conduite en 1983 par Olweus en Suède ${ }^{5}$. L'auteur montre d'abord que l'analyse des données semble valider l'idée que l'agression influence la performance scolaire. Cependant, la prise en compte dans l'analyse d'indicateurs du contexte familial annule complètement ce lien causal. Il apparaît ainsi que ce sont des antécédents à cette période scolaire qui expliquent à la fois le niveau des comportements répréhensibles et celui des résultats scolaires.

Penchons-nous alors vers les observations sur les premières années de scolarité. Hinschaw ${ }^{6}$ relève prudemment qu'il existerait un lien spécifique entre les troubles attentionnels et les performances en début de scolarité, en particulier dans l'apprentissage de la lecture. La relation entre agression et performance se développerait plus tard dans le cours de la scolarité. Ces résultats concordent avec l'analyse approfondie exposée par trois spécialistes reconnus dans un ouvrage documenté sur l'explication des comportements antisociaux des adolescents ${ }^{7}$. Ces auteurs montrent la persistance et la consistance de l'impact de l'hyperactivité ainsi que du style coercitif d'éducation parentale sur ces comportements. En résumé, l'ensemble de ces travaux semble montrer que la performance scolaire et les comportements agressifs seraient déterminés principalement par des déficiences cognitives et éducatives précoces.

À ce stade de notre réflexion, nous constatons que la relation directe entre la réussite scolaire et les comportements violents apparaît peu évidente. Comme le soulignent Tramontana, Hooper et Selzer ${ }^{8}$, à la suite d'une analyse de 74 études longitudinales, il manque une véritable perspec- 
tive théorique susceptible d'articuler le développement de l'enfant et son adaptation au contexte scolaire pour dégager des conclusions claires.

\section{L'agression est-elle générée par la frustration que crée l'échec scolaire?}

La théorie sur la frustration-agression développée en 1989 par Berkowitz ${ }^{9}$ devrait permettre de combler les lacunes théoriques que nous venons d'évoquer, même si elle est peu mise en ouvre dans les travaux sur la violence dans le cadre scolaire. L'idée intuitive que la frustration engendre des réactions violentes a une longue histoire ${ }^{10}$. Dans notre cas, il s'agit d'abord de se demander si un mauvais résultat scolaire, ou la menace d'un tel résultat, engendre de la frustration. Il faut pour cela que l'échec vienne contrecarrer un projet, ce qui implique que la performance scolaire apparaisse comme un objectif désirable pour l'enfant, objectif qu'il doit pouvoir atteindre pour maintenir ou renforcer l'estime qu'il a de lui-même. Dans ce cas, une mauvaise note va certainement engendrer une frustration et une réaction émotionnelle qui peut prendre différentes directions, comme la colère, ou la honte. La direction de la réaction émotionnelle dépend notamment de l'attribution de la cause de l'échec. Lorsque celle-ci désigne un autrui, un enseignant ou un camarade, l'élève va éprouver un ressentiment vers cet autrui, susceptible de dégénérer en acte d'hostilité lorsqu'il ne parvient pas à réguler ses émotions différemment. Cellesci peuvent prendre une autre coloration si elles sont dirigées contre soi lorsque l'élève s'estime responsable de son échec.

Décrit ainsi, le processus paraît clair. Cependant, il n'a pas donné lieu à une validation directe et il offre plutôt une interprétation de certains comportements individuels. En fait, il pose principalement deux problèmes qui sont associés à l'évolution historique de l'école. Les travaux réalisés avant les années 1980 se situaient dans un cadre scolaire qui était marqué par la réussite d'un petit nombre d'élèves promus à des études longues et des rôles sociaux dominants, comme ceux de Wyer et ses collègues ${ }^{11}$. Dans un tel cadre, la division des élèves selon leurs performances initiales était peu 
L'échec scoldire atteint davantage

l'estime de soi lorsque les élèves sont amenés à croire que l'obtention d'une position professionnelle valorisée dépend de leur propre volonté. problématique et peu contestée. Les réformes scolaires, lancées dès les années 1960 dans la plupart des pays européens, ont progressivement modifié cette situation, où la norme était en fait la relégation, pour le plus grand nombre, dans une voie peu exigeante. En prônant l'égalité des chances, les réformes ont inscrit la réussite comme la norme que tous peuvent atteindre, grâce à leurs capacités, tout en maintenant un système de sélection par le biais d'évaluations constantes. Dès lors, les élèves se trouvent soumis à une compétition plus forte orientée par l'obsession de la réussite. En effet, celle-ci devient le passage obligé à l'obtention d'une position professionnelle, et de plus les élèves sont amenés à croire qu'elle dépend de leur propre volonté. L'échec scolaire atteint ainsi plus profondément l'estime de soi, engendre une frustration plus précoce et des réactions émotionnelles plus intenses qu'elles ne l'étaient auparavant. L'évaluation devient une menace sérieuse dans le sens où ses conséquences apparaissent plus évidentes pour les élèves et leurs parents.

Par conséquent, le modèle de la frustration-agression semble plus opérant pour expliquer les liens actuels entre agression et performance scolaire, en suggérant que la causalité va davantage dans le sens de l'échec - ou de sa menace - vers la violence que l'inverse. Pourtant, il faut bien reconnaître que ce raisonnement théorique n'a reçu que peu de validations empiriques. Par exemple, nous avons conduit une étude sur les agressions dans le cadre scolaire auprès de près de 2500 adolescents en Suisse ${ }^{12}$. La performance scolaire individuelle n'intervient aucunement dans l'explication des actes violents. Il apparaît cependant des différences selon le regroupement des élèves dans des voies ou des filières différenciées selon leur niveau de performance avant leur passage au degré secondaire. Examinons donc plus en détail ce processus.

\section{La progression scolaire : la performance pour les uns, la confrontation pour les autres?}

Personne ne peut actuellement contester que l'adaptation scolaire durant les premières années dépend principalement 
de la conjugaison des ressources familiales, d'un fonctionnement psychologique équilibré et, bien entendu, d'un enseignement adéquat. Les problèmes causés par l'un et/ou l'autre de ces systèmes occasionnent des difficultés d'adaptation scolaire qui se manifestent souvent à la fois sur le plan des performances cognitives et des ajustements comportementaux. C'est sur la base de cette adaptation plus ou moins bonne que la sélection scolaire va opérer une répartition des élèves dans des voies ou des établissements plus ou moins prestigieux. Même lorsqu'elle intervient tardivement, la division qui en résulte favorise la progression des élèves qui bénéficient déjà du meilleur développement et entrave celle de ceux qui éprouvent des difficultés. La perspective théorique de Harris permet de bien comprendre comment se construit l'interaction entre performance scolaire et agression, non pas à un niveau individuel, mais à un niveau groupal $^{13}$. L'auteur propose une nouvelle théorie de la socialisation en articulant les résultats issus des approches génétiques et psychologiques du développement. Dans ce cadre, la socialisation découle principalement des groupes d'appartenance les plus significatifs pour les enfants. Si le groupe familial constitue l'environnement de base pour le développement initial, ce sont rapidement les pairs qui vont constituer le groupe de référence pour les enfants et les adolescents. De la langue aux comportements, l'influence de la famille apparaît plus faible que celui des groupes de pairs. Cette influence s'accentue à l'adolescence lorsque la construction de l'identité sociale prend appui sur la différenciation avec d'autres groupes, enfants, adultes ou autres groupes de pairs. L'école, et encore davantage la classe, représente un groupe naturel pour les élèves. De ce fait, ce groupe va être caractérisé par ses résultats scolaires comparés à ceux d'autres classes ou d'autres écoles. Il en découle aisément que l'identité et la réputation des adolescents vont dépendre largement de leur appartenance à un «bon » ou un « mauvais » groupe d'élèves $^{14}$. Comme Berkowitz ${ }^{15}$ le rappelle, l'appartenance à un groupe de perdants induit typiquement des réactions émotionnelles découlant de la frustration qui prennent la forme d'actes d'hostilité dirigés le plus souvent contre les membres de son propre groupe. Et nous observons en effet dans notre étude que les élèves relégués, suite à leur faible performance en primaire, dans les filières scolaires les plus basses, en majorité des garçons issus de l'immigration, commettent
Même lorsqu'elle

intervient

tardivement,

Ia division

qui résulte de

Ia sélection

scolaire favorise

la progression

des élèves qui

bénéficient déjà

du meilleur

développement

et entrave celle

de ceux qui

éprouvent des

difficultés. 


\section{L'intensification} de la sélection et de Ia compétition induit une dégradation du climat scolaire en amenant les exclus à commettre davantage d'actes de violence. davantage d'agressions que les bons élèves, agressions dirigées le plus souvent contre des garçons de leur propre groupe scolaire $^{16}$. Nous observons également que ces mêmes élèves se sentent davantage en insécurité dans leur école que les bons élèves, mais que cette différence disparaît à mesure qu'ils s'éloignent de l'établissement. Dans une même perspective, nous pouvons citer les résultats d'une étude de Felson, Liska, South et McNulty conduite auprès de plus de 2000 adolescents entre 15 et 17 ans provenant de 87 lycées américains ${ }^{17}$. Les auteurs montrent notamment, en comparant les établissements, que l'augmentation de la compétition académique augmente le niveau global de violence et de délinquance. En revanche, au niveau individuel, il existe un lien négatif entre la performance scolaire et la perpétration d'actes de violence. Ainsi, l'intensification de la sélection induit une dégradation du climat scolaire en amenant les exclus à commettre davantage d'actes de violence.

\section{Conclusion}

À la fin de ce cheminement dans une littérature touffue, nous pouvons mettre en évidence une dynamique caractérisée par deux mouvements. Dans un premier temps, l'enfant passe par une phase d'adaptation durant laquelle l'évaluation devrait permettre de relever les problèmes d'apprentissage et de comportements agressifs. L'évaluation devient une menace de marginalisation scolaire et exacerbe les problèmes comportementaux, si son objectif ne vise pas à trouver les moyens d'intégrer le jeune élève en difficulté. Dans un second temps, l'évaluation apparaît clairement pour l'élève comme un moyen de le disqualifier et elle induit une inquiétude qui pèse sur l'estime qu'il a de lui-même. La hiérarchisation des élèves qui en découle induit une compétition qui accentue la menace de l'échec et entraîne une dégradation du climat scolaire. Les comportements agressifs se développent au sein des groupes d'élèves inquiets et marginalisés sur le plan scolaire, dans des voies ou des établissements peu prestigieux. Lorsque la compétition s'accentue par le biais d'évaluations plus nombreuses et soutenues apparaissent également des réactions individuelles aux évaluations qui prennent la forme d'agressions face aux enseignants, dans certains cas, ou de désengagement scolaire dans d'autres, 
quels que soient les voies ou les établissements. La réponse à notre question initiale est donc plus complexe que prévue : certes, ce sont bien les élèves les plus faibles scolairement qui manifestent le plus d'hostilité face aux autres élèves, mais nous observons aussi une propension à l'agression, notamment face aux enseignants, parmi tous les élèves qui en quelque sorte contestent l'évaluation scolaire.

1. R.S. Wyer,

D.A. Weatherley,

G. Terrell, «Social roles, agression, and academic achievement », Journal of Personality and Social Psychology, 1965, $\mathrm{n}^{\circ} 1$, p. 645-649.

2. R.S. Wyer,

D.A. Weatherley,

G. Terrell, op. cit., p. 646.

3. Hirschi,

M.J. Hindelang,

« Intelligence and

delinquency: A

revisionist review ",

American Sociological

Review, 1977, n 42,

p. 571-587.

4. S.P. Hinschaw,

« Externalizing behavior problems and academic underachievement in childhood and adolescence: Causal relationships

and underlying

mechanisms ",

Psychological Bulletin, 1992, no 111, p. 127-155.

5. D. Olweus, «Low school achievement and aggressive behavior in adolescent boys ", in D. Magnusson (dir.), Human Development : An Interactional Perspective, San Diego, CA, Academic Press, 1983, p. 353-365.

6. S.P. Hinschaw, op. cit.

7. M. Rutter, H. Giller

\& A. Hagell, Antisocial Behavior by Young People, Cambridge, Cambridge University Press, 1998.

8. M.G. Tramontana, S.R. Hooper \& S.C. Selzer,
« Research on the preschool prediction of later academic achievement: A review », Developmental Review, 1988, no 8, p. 89-146.

9. L. Berkowitz,

«Frustration-aggression hypothesis:

Examination and reformulation ", Psychological Bulletin, $1989, \mathrm{n}^{\circ} 106$, p. 59-73.

10. F. Pahlavan, Les conduites agressives, Paris, Armand Colin, 2002.

11. R.S. Wyer,

D.A. Weatherley,

G. Terrell, op. cit.

12. A. Clémence,

F. Rochat, C. Cortolezzis, P. Dumont, M. Egloff, M. C. Kaiser, Scolarité et adolescence : les motifs de l'insécurité, Berne, Haupt, 2001.

13. J.R. Harris, Pourquoi nos enfants deviennent ce qu'ils sont, Paris, Laffont, 1999.

14. N. Emler, S. Reicher, S., Adolescence and Delinquency, Oxford, Blackwell, 1996.

15. L. Berkowitz, op. cit. 16. A. Clémence, F. Rochat, C. Cortolezzis, P. Dumont, M. Egloff, M., C. Kaiser, op cit. 17. R.B. Felson, A.E. Liska, S.J. South, T. McNulty, «The subculture of violence and delinquency: Individual vs. school context effects », Social Forces, 1994, n 73 , p. $155-173$. 